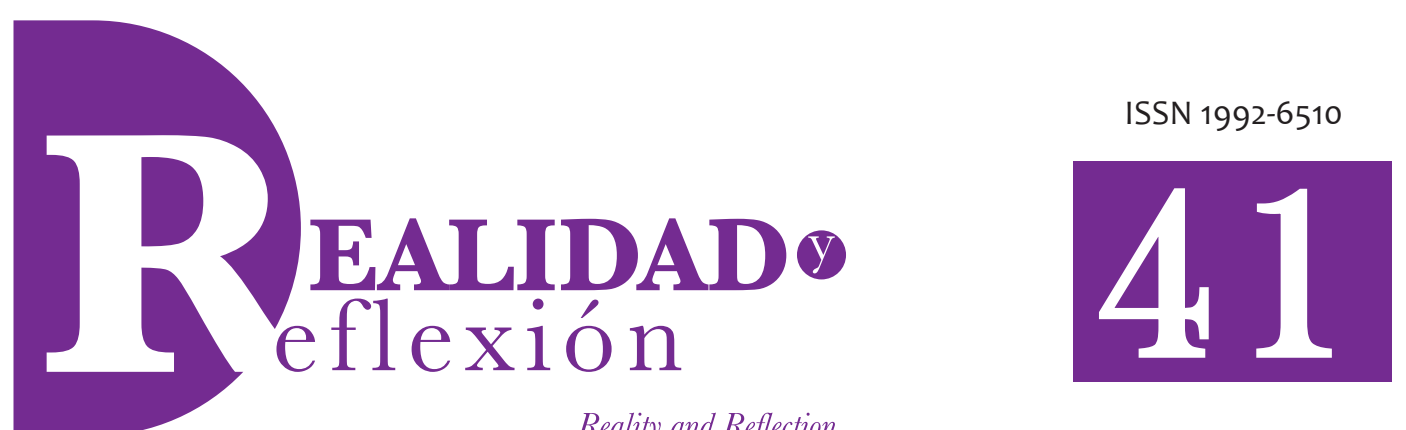

Reality and Reflection

año 15, N 41, San Salvador, El Salvador, Centroamérica Revista Semestral Enero-Junio 2015

Year 15, No 41, San Salvador, El Salvador, Central America Semestral Journal January-June 2015

\title{
Guía para el docente de la enseñanza del inglés como segunda lengua
}

\author{
Teacher's Guide to the teaching of English as a second language
}

\author{
Roberto Vladimir Carbajal \\ Licenciado en Educación, Especialidad idioma inglés, \\ Universidad de El Salvador. \\ Master en Investigación Social, \\ Universidad de El Salvador. \\ Docente e investigador de la Facultad de Ciencias Sociales \\ de la Universidad Francisco Gavidia.
}

\section{RESUMEN}

Se dice que la edad apropiada para el aprendizaje de una segunda lengua es durante la infancia, puesto que el nuevo hablante aprenderá la segunda lengua en un entorno natural y sencillo. Los niños no traducirán de un idioma a otro.

Este artículo se enfoca a los desafíos que enfrentará el maestro experto en la enseñanza a los niños y utilizar diferentes recursos con el fin de motivar al alumno a expresarse en el idioma de destino. El artículo presenta los métodos, las estrategias y los recursos que los profesores tienen que tomar en cuenta para el desarrollo de un programa que podrán aplicar en clase.

Es importante mencionar que el lenguaje es una acción comunicativa, y este nivel de aprendizaje de nuevo idioma es un momento para disfrutar, hablar y escuchar en esta edad en la que le niño está aprendiendo, pues se concibe que es la apropiada; así también el profesor juega un papel importante para crear junto con el alumno el ambiente propicio, los recursos, las herramientas y toda las estrategias necesarias. RECURSOS DE APRENDIZAJE, NIÑOS, NUEVO IDIOMA, MÉTODOS Y ESTRATEGIAS. 


\section{ABSTRACT}

It is said that the appropriate age for learning a second language is during infancy, since the new speaker learn the second language in a natural and simple environment. Children do not translate from one language to another.

This article focuses on the challenges facing the expert teacher in teaching children and use different resources in order to motivate students to speak in the target language. The article presents the methods, strategies and resources that teachers need to consider in developing a program that may apply in class.

It is noteworthy that language is a communicative action, and this level of learning new language is a time to enjoy talking and listening in this age when you child is learning, then it is conceivable that is appropriate; so the teacher plays an important role to create with the student the right environment, resources, tools and all the necessary strategies. LEARNING RESOURCES, CHILDREN, NEW LANGUAGE, METHODS AND STRATEGIES.

\section{Introducción}

Este texto o guía, basada en fundamentos prácticos, se ha planteado por la necesidad que existe de aprender un segundo idioma, en este caso el inglés, pero a temprana edad. La realidad que enfrenta el docente en este ámbito es que se encuentra con un terreno fértil y que hay que cultivar; por esta razón el maestro debe plantearse qué estrategias va a utilizar y los materiales y recursos necesarios que motiven al niño a expresarse en el idioma meta.

Los niños emplean estrategias en el aprendizaje de la lengua en un ambiente natural. Entre estas estrategias están la imitación, la repetición, el habla telegráfica y la incorporación. Los primeros intentos de producir habla son intentos de imitar palabras que emplean otros. En la medida en que incorporan significado, los niños siguen usando estas palabras y con frecuencia repitiéndolas. Luego, los niños comienzan a combinar palabras aprendidas en esta forma, demostrando creatividad en la producción de habla telegráfica.

Los niños son entusiastas, curiosos, interesados en el aprendizaje de una lengua. Los niños disfrutan del lenguaje con placer dominando el sistema articulatorio y empleando palabras poco familiares, muchas veces como si estuvieran experimentando sus experiencias. Cada palabra nueva, cada sonido diferente es una curiosidad nueva para ellos y la repetirán mil veces hasta aprenderla.

A esto podemos hacer una referencia general a algunas teorías sobre el aprendizaje del lenguaje del niño:

El lenguaje es un hecho social que, como tal, se desarrolla a través de las interacciones dentro de una comunidad. Según Lev Vygotsky, psicólogo soviético del siglo XX, la adquisición del lenguaje implica no solo la exposición del niño a las palabras, sino también un proceso interdependiente de crecimiento entre el pensamiento y el lenguaje. La influyente teoría de Vygotsky sobre la "zona de desarrollo próximo" afirma que los profesores deberían tomar en consideración el potencial de aprendizaje futuro del niño antes de intentar ampliar sus conocimientos.

Piaget destaca la prominencia racional del lenguaje y lo asume como uno de los diversos aspectos que integran la superestructura de 
la mente humana. El lenguaje es visto como un instrumento de la capacidad cognoscitiva y afectiva del individuo, lo que indica que el conocimiento lingüístico que el niño posee depende de su conocimiento del mundo. Su estudio y sus teorías se basan en las funciones que tendría el lenguaje en el niño. Para Piaget las frases dichas por los niños se clasifican en dos grandes grupo: las del lenguaje egocéntrico y las del lenguaje socializado; estas a su vez se dividen en las siguientes categorías: Lenguaje egocéntrico: Repetición o ecolalia, el monólogo, el monólogo colectivo; lenguaje socializado: La información adaptada.

\section{Condiciones de apoyo al aprendizaje en el salón de clases}

Para enseñar un idioma extranjero (en este caso el inglés) los maestros deben apoyarse en material didáctico como fichas (flash cards), afiches, murales que tengan que ver con el tema, juegos, etc. Si se va a enseñar la palabra "mamá" (mother) se muestra figuras de una familia, de muchas madres, etc.

\section{1 Énfasis en el significado más que en la forma}

Una interacción que tenga sentido en el salón de clase implica mucho más que la mera interacción lingüística y es indispensable crear condiciones para que estas se den y el significado se pueda negociar si se pretende que el niño progrese.

Un ejemplo de eso sería un juego. Los niños emplean un lenguaje simple en un contexto real y significativo.

Ejemplo: Se van a enseñar objetos de toda clase. Muchos objetos se meten en una bolsa, los niños están todos sentados en un círculo y empieza así:
- Carolyn: Will you give me a watch please?

- Marisol: Here you are

- Carolyn: Thank you.

Cuando ya se tiene el objeto se le pasa al niño de al lado.

- Susie: Will you give me a cat ?

- Carlos: Sorry, I don't have one. ** Como no hay cat, Susie debe pedir otra cosa hasta que consiga una que si hay.

- Susie : Will you give me a shoe?

- Carlos: Here you are...

Y así continua el juego con todos los otros niños.

En general, el aprendizaje del idioma inglés en esta etapa del niño se centra en un enfoque comunicativo con el fin de beneficiar su desarrollo integral, el cual se enlaza con cuatro dimensiones del desarrollo infantil:

- Dimensión social: El idioma inglés es un conocimiento de carácter social y por ende se aprende en sociedad, tanto la pronunciación como la entonación y el uso pragmático le da validez.

- Dimensión afectiva: el aprendizaje se favorece con la confianza y el afecto, la relación que el niño establece con el educador y demás compañeros, buscando la aceptación del grupo, lo cual es crucial para su apropiación.

- Dimensión física: El juego, más que una característica, es una definición del niño, 
los movimientos, desplazamientos y melodías en los juegos bilingües impulsan el desarrollo en forma determinante.

- Dimensión intelectual: El acercamiento al inglés se centra en la compresión de significados por ello hay que potenciar la construcción del pensamiento y no la simple traducción de palabras.

\subsection{El habla del maestro como apoyo al aprendizaje del niño}

Para que el niño tenga éxito en su aprendizaje los maestros de inglés deben hablarles en inglés fuera y dentro del salón. Es importante apoyar la comunicación con gestos, expresiones faciales y acciones, pues esto da la clave para entender el significado de lo que escuchan, familiarizándose con los sonidos, ritmos y acentos.

Para comenzar una actividad:

- Listen: Escuchen.

- Listen everyone: Escuchen todos.

- Are you ready?: ¿Estás listo?

- Say it with me: Dilo conmigo.

- Do this like me: Haz esto como yo.

- Follow me: Síganme.

- Open your books at page 5: Abran sus libros en la página 5.

- Turn to page 10: Vayan a la página 10.

- Colour the picture: Pinta la figura.
- Copy this: Copia esto.

- Write this in your notebook: Escribe esto en tu cuaderno.

\section{3 ¿Qué contenidos de inglés se pueden dar en preescolar?}

- Me (name, boy, girl, things we do everyday).

- School (pen, pencil, crayons, paper, table, chair, scissors, glue, window, door, chalkboard, folders).

- Colors (red, blue, yellow, green, purple, orange, brown, black, pink, white, gray).

- Shapes (circle, square, triangle, rectangle, oval).

- Family (father, mother, sister, brother, grandmother, grandfather, uncle, aunt, nephew, niece).

- Transportation and communication (car, bicycle, motocycle, boat, airplane, helicopter, television, telephone, radio, newspaper,).

- Numbers.

- Vowels (a, e, i, o, u).

- Fruits (apple, pear, orange, grapes, bananas).

- Food (carrots, beans, ice cream, milk, water etc.).

- My body parts (head, eyes, ears, nose, mouth, shoulders, hair, neck, arms, hands, fingers, legs, feet, toes etc.). 
- Animals (farm animals, wild animals, dog, cat, bird, mouse, etc.).

- Especial dates (Valentine's Day, Christmas, Mothers Day, Father's Day).

- Opposites (big-small, outside-inside, full-empty, up-down, long, short, cold, hot, etc.).

- Summer, Winter, Spring, Fall.

- Community helpers (doctor, teacher, fireman, policeman, mailman).

\section{Estrategias de aprendizaje}

\subsection{Desarrollo del vocabulario Speaking and Listening}

Analizaremos la semántica desde el punto de vista del desarrollo del vocabulario infantil.

La mayoría de los estudios acerca del desarrollo del vocabulario de los niños en el momento de su ingreso a la escuela (a los tres años) se produce un incremento rápido del vocabulario, incremento que es mucho mayor que lo que ocurrirá posteriormente, llegando a tener un promedio de 896 palabras, y a los tres años y medio 1222 palabras (Smith, 1980), y lo incrementan a una velocidad de alrededor de 1000 palabras por año, en las etapas de la primaria, y de 2000 en la secundaria, por año esto; es lo que sostiene Harris (1966).

Sin embargo, Brooks (1970) sostiene que al iniciar la escolaridad los niños tienen un promedio de 8000 palabras e incluso pueden dominar hasta 20,000.

Entonces surge la pregunta: ¿Cómo se aprenden las palabras? No existe, aparentemente, un modelo rígido de aprendizaje; este varia correlativamente con el desarrollo intelectual. Al parecer, en un principio, la palabra servirá para indicar una categoría de objetos que uno en especial. Generalmente, el niño es atraído por una determinada característica del objeto, a la cual denomina y le sirve de etiqueta o rótulo para designar los objetos que posee esa misma característica. Por ejemplo, el uso del término "tick tack" para indicar un reloj.

El aprendizaje de palabras, en una etapa superior, implica dos procesos. En el primero el niño asocia las unidades dentro de contextos que le llegan a ser familiares, es decir habría una emisión activa. El segundo proceso implica individualización de unidades semánticas a partir de contextos escuchados; es decir, este proceso se realiza por la asimilación de enunciados propuestos por los adultos. La experiencia muestra que los niños, a partir de cierta edad, aprenden también palabras preguntando a los adultos el nombre de las cosas.

A continuación se presenta un plan de actividades de desarrollo del vocabulario infantil, sobre la base de La expresión oral y las destrezas de escuchar.

\subsection{Expresión oral}

La forma más simple y directa de desarrollar el lenguaje es facilitar las oportunidades para que hable y hable sin trabas. Así, servirán para este objetivo todas las actividades y experiencias que proporcionen nuevos significados o que refuercen con distintos matices los ya adquiridos. Nuevas experiencias llevaran nuevos significados y nuevas palabras. 
A continuación se sugieren actividades verbales efectuadas sobre la base de un contexto (situación) que constituye la fuente de la comunicación entre los niños y el maestro.

Por sus características de autenticidad, brevedad, repetición, musicalidad y ritmo, las nursery rhymes, riddles, songs and limerick son de gran potencialidad didáctica en nuestras aulas de educación infantil y tienen la ventaja de que los alumnos las memorizan de forma lúdica y rápida, repitiéndolas y utilizándolas significativamente casi sin la intervención del profesor (adquiriendo así no sólo vocabulario y estructuras, sino también ritmo, entonación y pronunciación).

-Canciones, “chants” y cuentos:

Motivan a los niños, ayudan a crear un ambiente agradable para el aprendizaje y tienen mucha repetición, lo que facilita la comprensión y la memorización. En cuanto a la producción oral, se crean situaciones donde los niños van produciendo palabras y expresiones (Ejemplos: Good Morning!, Hello!, Happy Birthday, etc.) participando en diálogos, en las canciones y los cuentos.

\section{-Actividades TPR ("Total Physical Response") y juegos:}

Estas actividades contribuyen a desarrollar la comprensión oral, ya que las instrucciones que va diciendo el profesor son en inglés. Al mismo tiempo, los niños participan y se divierten mucho.

Las actividades TPR siguen el método "Total Physical Response": la maestra ofrece una serie de instrucciones y los niños responden de forma no verbal realizando acciones (o mímica) apropiadas.
El juego es el método y el recurso didáctico por excelencia en la educación infantil, que unido a la dramatización de cuentos, canciones, etc., es una parte muy importante porque permite a los alumnos poner en práctica el lenguaje aprendido. Proporcionan una buena oportunidad para que los alumnos no sólo practiquen palabras sueltas, sino también expresiones y frases.

Además son necesarios los soportes visuales de aprendizaje periférico, tales como flashcards, pictograms, posters, drawings ... que ayudan al niño a reconocer la imagen visual de las expresiones que esté trabajando y así aprender de una forma natural como su lengua materna (Natural approach).

\section{Métodos de enseñanza-aprendizaje apropiados para la adquisición de una segunda lengua en el aula de educación infantil}

Los métodos más apropiados para la educación infantil son estos: The Silent Way, the Community Language Learning, the Natural Approach, the Total Physical Response y The Suggestopedia.

\subsection{The Silent Way}

Su creador fue el profesor Caleb Gattegno. Entre sus características subrayamos las siguientes:

- No se usa la lengua materna.

- El aprendizaje se produce a través de pósteres, tarjetas con sonidos o morfemas codificados por colores y cuisiniere rods (palitos de madera de diferente tamaño y color), porque el aprendizaje mejora 
cuando intervienen objetos que el alumno puede manipular. Además, los recursos visuales se consideran mediadores asociativos.

- Se refuerza el sonido, la precisión y la entonación.

- El profesor permanece en silencio la mayor parte del tiempo, con la finalidad de que sean los alumnos quienes hablen y practiquen la mayor parte del tiempo. En cambio, ejerce un gran control de todo el proceso y utiliza la comunicación no verbal.

- El silencio es importante porque la imagen acústica tarda algunos segundos en grabarse en la mente, además permite la concentración del alumno y la organización mental de los datos lingüísticos recibidos.

- Basado en la teoría estructuralista de la lengua, diseña su silabeo en torno a estructuras lingüísticas y vocabulario.

- El procedimiento podría ser este: el profesor establece la situación y centra al alumnado en alguna estructura que él produce. Los alumnos responden a señales visuales repitiendo y poco a poco producen mensajes lingüísticos cada vez más complejos. Finalmente, se pone en común lo sucedido.

\subsection{The Community Language Learning}

Derivado del Enfoque Humanista, surge como una aplicación del Counselling Learning. Fue desarrollado por el psicólogo Charles Curran, aplicando al aprendizaje (en este caso de un idioma) las técnicas que usaba en su consulta psicológica. Algunas de sus características son estas:

- La lengua es un sistema de comunicación social.

- El aprendizaje afecta a la personalidad de forma global.

- El profesor actúa como consejero, amigo, tutor o recurso y es responsable de crear un ambiente en el que todos se sientan seguros.

- Los alumnos son, en primer lugar, miembros de una comunidad que aprenden mediante la interacción, y que deben, entre otros, apoyar a los otros miembros del grupo y comunicar sus sentimientos sin reparo.

- No existe un silabeo definido, sino que éste se va formando a partir de las necesidades comunicativas del grupo.

- El procedimiento podría ser en círculo, con el profesor fuera, un alumno le dice algo al profesor, quien se lo devuelve en la FL. El alumno lo repite y se le graba. Cuando se ha terminado la "ronda", se sientan en semicírculo con el profesor delante, se escucha la cinta y se escriben las oraciones en la pizarra. A continuación se analizan y se ponen ejercicios.

\subsection{The Natural Approach}

Sus autores son Stephen Krashen y Tracy Terell, quienes basan sus estudios y sus posteriores conclusiones en la diferenciación entre aprendizaje y 
adquisición.

El aprendizaje de una FL tiene que parecerse más a la adquisición de la L1. Ésta se va a adquirir mediante la simple exposición del niño al lenguaje que se produce a su alrededor (input). Su habilidad natural para usar el lenguaje es el resultado de muchos procesos subconscientes.

Según Krashen, el lenguaje que se "aprende" y el lenguaje que se "adquiere" quedan almacenados en compartimentos separados del cerebro, existiendo muy poca comunicación entre ambos. El aprendizaje no ayuda directamente a la adquisición. A pesar de ello, el compartimento de la adquisición puede verse beneficiado considerablemente por la función monitora del compartimento del aprendizaje (detectando y corrigiendo posibles errores tanto durante la producción como en la recepción). Por ello es importante mantener un equilibrio adecuado entre dosis de exposición al lenguaje (adquisición) y las de estudio reflexivo (aprendizaje).

Otra de las bases sobre las que se diseña este enfoque se refiere al input y al filtro afectivo. Se entienden como los dos verdaderos factores que influyen en el aprendizaje, más que la edad, la aptitud, la calidad de la enseñanza o el tiempo de exposición a la nueva lengua.

El input debe tener características para que sea el adecuado. Debe contener lenguaje que el alumno ya conoce y otro que no ha visto nunca, por tanto debe ser de un nivel un poco más alto de lo que es capaz de usar, pero a un nivel que sea capaz de entenderlo (roughly-tuned input), igual al lenguaje simplificado que utilizan los padres con sus hijos, slightly above their level. Así, los estudiantes pueden adquirir solos el lenguaje siempre que se les dé una buena cantidad de input comprensible, y, como es lógico, dentro de experiencias en las que este input sea adecuado (contexto).

En cuanto al filtro afectivo, es decir, la actitud o disposición de ánimo con que el individuo actúa o responde a los mensajes que recibe, Krashen afirma que éste facilita u obstaculiza la adquisición. Un filtro alto lo hace prácticamente imposible, y a la inversa. Por ello, en un ambiente de relajación y de afecto y comprensión, el individuo se encuentra en inmejorables condiciones para asimilar con mayor eficacia y rapidez dicho input.

Las técnicas y actividades propuestas por este enfoque no ofrecen novedades importantes. Son muy parecidas a las de los métodos anteriores: enfoque comunicativo, método situacional.

Teoría lingüística sobre la que se basa este enfoque: lengua como un instrumento para la expresión de significados. Las reglas no son importantes para establecer relaciones entre palabra y significado.

Teoría sobre el aprendizaje de la lengua: es un proceso inconsciente, que se activa al implicarse el sujeto en actos de comunicación.

Diseño: se trabajan las cuatro destrezas en dos áreas, basic personal communicative skills (adquisición) y academic learning (aprendizaje).

Sílabo: son unas actividades comunicativas, seleccionadas y graduadas. Centros de interés seleccionados por los alumnos.

Funciones del profesor: diseñar y presentar 
las actividades comunicativas, aportar gran parte del comprehensible input y crear un clima motivador y sin tensiones.

Uso de materiales auténticos que envuelven al alumno en una situación comunicativa. Procedimientos: situaciones de comunicación y uso comunicativo de la lengua, prestándose atención al significado, no a la forma.

\subsection{Total Physical Response}

Técnica desarrollada por el psicólogo americano James Asher, basada principalmente en la coordinación entre el lenguaje y la acción, dándole gran importancia a la actividad física. Según su teoría, la adquisición de una lengua extranjera sigue un proceso paralelo al de la materna en su etapa infantil. Primero los niños reponden físicamente a las órdenes e instrucciones de sus padres $y$, posteriormente, lo hacen de forma oral. Se pone, así, un gran énfasis en las habilidades de comprensión así como en los aspectos afectivos y emocionales, tomados de la psicología humanista.

La visión que este método tiene de la lengua es la de un conjunto de estructuras que pueden realizar diferentes funciones comunicativas.

Tres condiciones facilitan o dificultan el aprendizaje de una FL:

1. Que siga un proceso parecido al de L1, en el que el niño desarrolla la destreza receptiva antes que la productiva, y esta última se desarrolla como resultado de la respuesta física a las instrucciones y órdenes de los padres. El habla surge, finalmente, de forma natural.
2. Al relacionar el lenguaje con la actividad física se consigue una actividad de los dos hemisferios, lo cual supone que existen unas funciones de aprendizaje distintas para el derecho y el izquierdo.

3. El estrés es un filtro afectivo que puede ralentizar o acelerar el aprendizaje, de manera que mientras más bajo sea el nivel de estrés más alto será el aprendizaje. Al respetar el período de silencio sin forzar la producción, disminuimos la ansiedad.

Aunque no existe un sílabo especialmente diseñado para este método, se basa en una visión estructural del lenguaje.

La actividad básica de clase la forman los ejercicios tipo drill (repetición), en los que se emplea el modo imperativo.

La influencia del alumno en los contenidos de aprendizaje es casi nula y su papel es el de escuchar y responder físicamente tanto de forma individual como de forma colectiva. El profesor, en cambio, es el elemento activo y director del proceso. Debe controlar el input que reciben e imitar el papel de los padres en el aprendizaje del primer idioma, mostrándose tolerante con los errores en las primeras fases $y$ disminuyendo la tolerancia conforme se va avanzando en la adquisición.

No existen unos materiales específicos para el desarrollo de este método. En las primeras etapas, la voz y los gestos del profesor son suficientes. Más adelante pueden utilizarse los elementos propios de la clase, como lápices, libretas, muebles, etc., y, con posterioridad, se pueden introducir también objetos reales y dibujos. 


\subsection{Suggestopedia}

Su creador fue el psiquiatra búlgaro George Lozanov, quien afirma que la razón de nuestra ineficacia a la hora de aprender una lengua extranjera radica en las barreras psicológicas que establecemos al aprender (inhibición). Por tanto, facilitaremos dicho aprendizaje si proporcionamos una atmósfera cómoda y relajada (mobiliario, interacciones, cambio de "personalidad", música...). La comunicación se produce a dos niveles, el consciente y el subconsciente $y$, por tanto, debemos atender ambos.

Procedimiento didáctico:

- Fase receptiva: el profesor lee el diálogo en FL (que está traducido a L1 en la misma página), junto a una música de fondo y los alumnos lo siguen. En una segunda lectura, los alumnos se relajan.

- Fase de activación: los alumnos trabajan en distintas actividades (dramatizaciones, juegos, canciones, preguntas y respuestas...).

Es importante que los alumnos asuman una personalidad nueva, con nuevo nombre, profesión... ya que aumenta la seguridad y disminuye la inhibición.

- Profesor: autoridad, pero para crear situaciones en las que el estudiante es más fácilmente sugestionable. Se necesitan unas cualidades personales y un entrenamiento especial para dirigir una clase de este tipo.

- Alumno: confían y respetan al profesor (“dejarse llevar").
Técnicas:

- Creación de un ambiente relajado (decorado, mobiliario, ...).

- Visualización.

- Aprendizaje periférico (el estudiante puede aprender de todo lo que le rodea, incluso si no dirige su atención a ello).

- Sugestión positiva.

- Elección de una nueva identidad.

- Role-play.

- Uso de música.

- Cantar, bailar, jugar...

V. Elementos básicos que debe recoger una programación de aula de Inglés en educación infantil

\subsection{La programación de aula deberá recoger los aspectos siguientes:}

La programación de aula es una unidad de trabajo relativa a un proceso completo de aprendizaje - enseñanza que no tiene duración temporal fija y en la cual se precisan el conjunto de objetivos didácticos, bloques de contenidos y actividades de aprendizaje y evaluación, metodología específica, orientaciones, relaciones de comunicación y organización de la clase y de las actividades, medios, recursos y materiales.

Los elementos mínimos que debe tener una programación son los siguientes: 
1. Objetivos (Generales y didácticos: Identidad, medio físico, comunicación).

2. Contenidos (separados en ámbitos de identidad, medio físico, comunicación).

3. Estrategias metodológicas (criterios y organización del aula).

4. Actividades (criterios de secuenciación de actividades; actividades de conocimiento previo, de desarrollo, de probación, consolidación y refuerzo, de evaluación).

5. Evaluación (qué, quién, cómo y cuándo evaluar).

6. Tratamiento de los temas transversales.

7. Atención a la diversidad.

8. Orientación y tutoría.

Dicho de otra forma: la UD debe tener estos cuatro aspectos:

1. Definición (tipo de centro, entorno físico y sociocultural, centro de interés, área, temporalización).

2. Fundamentación científica (fundamentos psicopedagógicos, fundamentos epistemológicos y sociológicos.

3. Desarrollo de la unidad (objetivos, contenidos, metodología, actividades...).

4. Evaluación.

\subsection{Actividades sugeridas}

Discriminación auditiva.
Diversos juegos en los que participan profesorado y alumnado.

Expresiones: boy / girl.

- El profesor llama a un alumno al centro del aula para que los demás lo vean y, al mismo tiempo que lo señala, le dice: boy

Después llama a una alumna al centro del aula $\mathrm{y}$, al mismo tiempo que la señala, le dice: girl.

- Relacionar o unir estas palabras con las pertenecientes al vocabulario de unidades anteriores.

Hello, boy.

Goodbye, girl.

How are you, boy?

Preguntas. Salen varios niños y niñas y el profesor pregunta: boy o girl? El resto del alumnado contesta.

Demostrativos: this is a... / this is an...

Nota: Utilizar el artículo “a” cuando la palabra siguiente comienza por consonante y "an" cuando comienza por vocal.

1. El profesor señala un niño y dice: This is a boy. Luego señala a una niña y dice: This is a girl.

Repite este ejercicio hasta que el alumnado lo comprenda. A continuación, niños y niñas señalan y nombran.

2. Realizar lo mismo que en el apartado 2.1, utilizando fotografías o entre los mismos alumnos. 
Cuando acierten se aplaude y si no aciertan, se hace ruido sobre las mesas.

Interrogaciones: what's this?

1. El profesor señala un objeto cercano o conocido y pregunta muy expresivamente: What's this?

Si los niños no responden, se insiste varias veces en la pregunta. Finalmente, responde con gran seguridad:

This is a boy / This is a girl.

Se repite con diferentes niños y niñas.

2. Cuando se haya comprendido, ellos formulan las preguntas y se responden entre ellos.

Para establecer turnos se puede recurrir a cualquier juego trabajado anteriormente.

\section{Números: one}

1. El profesor dice "one" mientras levanta un dedo. El alumnado repite.

Después dice "one" mientras enseña una tarjeta en la que está escrito el número uno.

2. A continuación se dirige a un niño o a una niña $y$, señalando un lugar donde hay varios objetos, como un bote de lápices, dice: Please, one.

Thank you.

Esta actividad pueden realizarla niños dando la misma orden a los otros que tienen que ejecutarla.
Incorporación del juego.

El juego se ha considerado en la historia de la humanidad como una actividad espontánea del niño. Es el niño el que juega, es el niño el que transforma su hacer de cada día en juego. En el niño no se distingue el juego del trabajo, todo para él es actividad, que puede ser sin duda, placentera.

En las actividades educativas que el niño realiza en la escuela y en las ocupaciones en las que entretiene la vida cotidiana en el hogar, puede considerarse el juego como una ocupación infantil que no tiene un fin distinto de sí misma, como en una acción que se realiza por sí misma y con ella se pretende simplemente jugar, el niño organiza su actividad, dispone de su tiempo y lo ocupa con sus juegos, con sus movimientos, con sus ocupaciones.

El placer del juego es inseparable del propio placer que constituye el hecho de jugar. Por lo general, se considera juego toda actividad lúdica que comporta un fin en sí misma, se juega por jugar.

A continuación se presentan algunas estrategias con base en juegos que se pueden desarrollar junto con los niños:

1. El uso del lenguaje: De esta forma entendemos que puede haber:

- Juegos no verbales que ayudan a conocerse mejor, a fomentar el espíritu lúdico y grupal, a "romper el hielo" y generar dinámicas de grupo; pero en los que también podemos trabajar conceptos, procedimientos y actitudes mediante técnicas que tan sólo exigen respuestas físicas (T.P.R.). 
Ejemplos de éstos pudieran ser "El nudo humano", "La granja”... o cualquier otro tradicional previamente adaptado.

- Juegos precomunicativos o facilitadores, que no pretenden específicamente la comunicación sino que trabajan sobre la lengua en distintos aspectos.

Ejemplos de éstos pudieran ser: En gran grupo, "Contamos los números de 5 en 5", "Decimos colores", etc.

Conviene recordar que al hablar de juegos lo haremos desde la concepción de que lo más importante es lo que denominamos conducta lúdica, o ese espíritu que ha de presidir nuestra intervención. De otra parte, sería inútil tratar de realizar una extensa nómina de juegos, pues cada uno de éstos serviría para una cosa u otra, dependiendo del enfoque que le aportemos.

- Juegos comunicativos, o aquellos que ponen el énfasis en la comunicación y desarrollan la fluidez. El éxito del juego no dependerá de la producción correcta de una estructura, sino de establecer convenientemente la comunicación, empleando todas las estrategias y subdestrezas necesarias. A menudo en ellos encontraremos el principio de "Information gap".

Ejemplos de éstos podrían ser: "Nos presentamos", “Losmensajes telefónicos”... o cualquier otro con estructura similar.

2. Las actitudes que se trabajen. Desde este punto de vista distinguimos fundamentalmente entre:
- Juegos cooperativos, o aquellos en los que sólo se busca el trabajo de valores positivos y desarrollo común muy relacionados con los temas transversales.

No hay ganadores, pues han sido creados para no generar dinámicas competitivas.

Ejemplo de éstos pudieran ser cualesquiera juegos debidamente reestructurados (pensemos en "Las sillas musicales", sin necesidad de que nadie quede eliminado.

- Juegos competitivos, o aquellos en los que los alumnos juegan para conseguir ganar. En este caso, tomando en consideración la fuente psicológica del currículum, nos daremos cuenta de que resultan habituales a estas edades escolares. Lejos de ser dogmáticos en este sentido, habremos de aprovechar estas tendencias para incentivar los valores no agresivos (no todo es válido para ganar) y tolerantes (hay que saber perder). Estará en nosotros nuevamente la focalización de cada juego, pues el más inocente de los juegos -mal interpretado- puede resultar humillante o viceversa.

De cualquier forma, habremos de dosificar mucho este tipo de juegos si en verdad creemos necesario atender a la diversidad de forma efectiva y real.

3. La técnica que se emplea. Así entendemos que desde este criterio puede haber:

- Juegos de adivinar

- Juegos de completar 
- Juegos de intercambiar

- Juegos de simular

- Juegos de solucionar problemas

- Juegos de "information-gap"

4. El medio que utilicemos. De ello entendemos que hay:

- Juegos con el cuerpo, o aquellos en los que éste es el elemento primordial. Implican movimiento, mímica, representaciones, etc.

- Juegos de tablero, o aquellos que independientemente del aspecto que se trabaje no utilizan otro espacio que el reducido a una mesa. Pueden ser de pareja o grupales, permitiendo al alumno la invención del mismo con materiales muy simples (dados, fichas, etc.).

- Juegos verbales o aquellos que van a requerir tan sólo la comunicación tanto oral como escrita. Aunque de alguna manera siempre llevarán implícito el apoyo gestual o quizás la escritura, nos referimos aquí a un tipo de juegos en los que la comunicación es el instrumento por excelencia.

Ejemplo: cualquier "Information-Gap" (repetición)

5. El aspecto lingüístico que se trabaje. Así, decimos que un juego puede ser:

- De vocabulario, en los que tratamos de reforzar, aprender o reciclar términos léxicos en terrenos tan diversos como la pronunciación, la entonación, el significado, etc.

Ejemplos de éstos pueden ser los clásicos "Bingo", "Word chain", "I spy with my little eye", "Fill in the gap", "Scrabble", etc.

- De estructuras, o aquellos que, con igual fin a los anteriores, se centran en unidades más amplias.

Ejemplos de éstos, pueden ser los ya mencionados, con sus consiguientes variaciones.

Por último, y previa a la finalización de este apartado, resultaría conveniente referirse a un asunto que por su interés, bien merece una mención especial: los agrupamientos. Independientemente del aspecto que consideremos al clasificar cualquier juego, éste llevará un tipo u otro de agrupamiento en el que también será útil realizar nuestras reflexiones.

La primera y más importante radicará en que debemos ser nosotros quienes pongamos cómo agruparnos para cada ocasión, estando esto derivado de la propia dinámica que ya utilizan nuestros alumnos y en la que sólo prevalece la idea de no compartir y ganar.

Eliminar estas tendencias formará parte de nuestro trabajo, y para ello también habremos de utilizar desde el inicio técnicas aleatorias de formación de grupos (números, tarjetas de colores, juegos asociativos, etc.) u otras en las que sea nuestro criterio objetivo el que prevalezca y no otro.

Artículo recibido: 15 de mayo de 2014 Artículo aprobado: 2 de julio 2014 


\section{Bibliografía}

GARDENER. B y GARDENER, F (2000): Classroom English Oxford. OUP.

HEARN IZABELLA, GARCÉS RODRÍGUEZ ANTONIO (2003): Didáctica del Idioma Inglés. (Madrid) Pearson Prentice Hall.

MONTIJANO CABRERA. M.P. (2001): Claves Didácticas para la enseñanza de la lengua extrajera. Archidona (Málaga). Aljibe

MOON , J ( 2000): Children Learning English. Oxford: MacMillan Heinemann.
ROTH, G (1998): Teaching very young Children .London: Richmond.

\section{Sitios web}

www.siteforteachers.com

www.webguideforteachers.com

www.abcteach.com 